\title{
EXPERIENCIAS DE INNOVACIÓN EDUCATIVA
}

- TOMO 1 -

GRANCOLOMBIANO

INSTITUCIÓN UNIVERSITARIA 


\title{
EXPERIENCIAS DE INNOVACIÓN EDUCATIVA
}

\author{
Autores \\ Luis Martín Trujillo Flórez \\ Ronald Mauricio Martínez Contreras \\ Hernando Espitia López \\ Juan Carlos Rojas Paredes \\ Yolanda Rocío Vargas Leguizamón \\ Germán Andrés Castro Cabal
}

Laboratorio de Innovación Pedagógica de Educación Virtual - LIPEV.

Dirección Académica de Educación Virtual

Vicerrectoría Académica.

Institución Universitaria Politécnico Grancolombiano

2017 


\section{POLI \\ POLITÉCNICO \\ GRANCOLOMBIANO \\ INSTITUCIÓN UNIVERSITARIA}

(c) Politécnico Grancolombiano

Experiencias de Innovación educativa

Primera Edición: Abril de 2018

ISBN: 978-958-8721-77-4

E-ISBN: 978-958-8721-79-8

Ebook: 978-958-8721-78-1

\section{Publicaciones Politécnico Grancolombiano}

Calle 57 \# 3-00 Este

Tel: 7455555, ext. 1171

E-mail: editorial@poligran.edu.co Bogotá, Colombia.

\section{Autores}

Luis Martín Trujillo Flórez

Ronald Mauricio Martínez Contreras

Hernando Espitia López

Juan Carlos Rojas Paredes

Yolanda Rocío Vargas Leguizamón

Germán Andrés Castro Cabal

\section{Lider de Publicaciones}

Eduardo Norman Acevedo

\section{Analista de Producción Editorial}

Paulo Mora Noguera

Ilustraciones

Mónica Nayibet Carrero Becerra

Diseño y Diagramación

Mónica Nayibet Carrero Becerra

Leonardo Stiglich Campos

\section{Corrección de Estilo}

Ana Ximena Oliveros

\section{Impresión}

Xpress Estudio Gráfico y Digital S.A.

\section{Impreso en Colombia}

Printed in Colombia
Trujillo Flórez, Luis Martín

Experiencias de innovación educativa / Luis Martín Trujillo Flórez; Ronald Mauricio Martínez Contreras; Hernando Espitia López; Juan Carlos Rojas Paredes; Yolanda Rocío Vargas Leguizamón; Germán Andrés Castro Cabal; Laboratorio de innovación educativa de educación virtual ; líder de publicaciones, Eduardo Norman Acevedo; analista de producción editorial, Paulo Mora Noguera; - Bogotá D.C.: Publicaciones Politécnico Grancolombiano., 2018.

170 p. : il.; $17 \times 24 \mathrm{~cm}$.

Incluye referencias bibliográficas.

ISBN: 978-958-8721-77-4

E-ISBN: 978-958-8721-79-8

Ebook: 978-958-8721-78-1

1. Innovación educativa 2. Software educativo 3. Simuladores 4. Modelos de simulación 5. Métodos de aprendizaje I. Norman Acevedo, Eduardo II. Mora Noguera, Paulo III. Laboratorio de innovación educativa de educación virtual IV. Institución Universitaria Politécnico Grancolombiano V. Tít.

SCDD 378.17 T866 Vo.1

Co-BolUP

Sistema Nacional de Bibliotecas - SISNAB Institución Universitaria Politécnico Grancolombiano

La Editorial Politécnico Grancolombiano pertenece a la Asociación de Editoriales Universitarias de Colombia ASEUC.

El contenido de esta publicación se puede citar o reproducir con propósitos académicos siempre y cuando se de la fuente o procedencia. Las opiniones expresadas son responsabilidad exclusiva de los autores.

\section{¿Cómo citar este libro?}

Trujillo et al. (2018), Experiencias de Innovación educativa, Bogotá: Editorial Politécnico Grancolombiano. 


\section{TABLA DE CONTENIDO}

\section{- Presentación de los autores}

- Introducción: La importancia de un Laboratorio de Innovación Pedagógica en Educación Virtual para la Institución Universitaria Politécnico Grancolombiano. Luis Martin Trujillo Flórez.

- Capítulo 1: Simulador de Diagnostico Empresarial. Ronald Mauricio Martínez Contreras y Luis Martín Trujillo Flórez.

- Capítulo 2: Simulador de análisis prospectivo. Ronald Mauricio Martínez Contreras y Luis Martín Trujillo Flórez.

- Capítulo 3: Simulador de gestión financiera para Administración Financiera. Hernando Espitia López y Luis Martín Trujillo Flórez.

- Capítulo 4: Simulador de inversión en Renta Variable para el Mercado de Capitales. Juan Carlos Rojas Paredes y Luis Martín Trujillo Flórez.

- Capítulo 5: Simulador de evaluación financiera de proyectos. Yolanda Rocío Vargas Leguizamón y Luis Martín Trujillo Flórez.

- Capítulo 6: Juego Gerencial basado en Balanced Scorecard. Germán Andrés Castro Cabal y Luis Martín Trujillo Flórez. 


\section{PRESENTACIÓN}

La Institución Universitaria Politécnico Grancolombiano pendiente de los múltiples cambios que está viviendo la educación y de los avances vertiginosos que tienen los ambientes virtuales ha decidido implementar un Laboratorio de innovación pedagógica e Investigación de Educación Virtual, el cual tiene como propósito gestionar con los docentes de la institución el desarrollo de proyectos de innovación centrada en estrategias de aprendizaje tales como: simuladores, juegos educativos o juegos serios, aplicaciones, realidad aumentada, realidad virtual, entre otros. El Laboratorio apoya todas aquellas iniciativas que conduzcan a la construcción de diversos ambientes educativos mediados por tecnología que les permitan a los estudiantes fortalecer la adquisición de competencias en los diferentes programas académicos.

Este proyecto se inicia en el 2017, es transversal a todas las facultades del Politécnico Grancolombiano y tiene como misión crear, gestionar, orientar, acompañar y diseñar con las entidades académicas cada uno de los proyectos considerados como innovadores, para ello las Facultades a través de los Departamentos Académicos proponen los diferentes proyectos a implementar, se hace la selección de estos y el Laboratorio orienta la planeación y construcción de cada uno de ellos.

Un área de innovación educativa debe tener un fuerte componente de investigación, por eso, además de implementar cada uno de los proyectos, estos se acompañan de un desarrollo investigativo que se trabaja en dos partes. La primera parte está compuesta por las memorias de las experiencias académicas que nos cuentan qué se hizo y cómo fue todo el proceso de construcción de cada proyecto de innovación. Este es el documento que usted lee en este momento y que nosotros presentamos con todo el cariño y esfuerzo con que realizamos nuestros proyectos. Aquí resumimos el arduo trabajo que se realizó durante todo el año que abarca desde la planeación, diseño, diagramación, redacción de guiones, diseño gráfico, animación, programación, y que continúa con las pruebas pilotos e implementación en las aulas con los estudiantes. 
El segundo componente investigativo consta de un artículo de investigación con la coautoría y direccionamiento del Observatorio de Educación Virtual del Politécnico Grancolombiano que junto con el Laboratorio de innovación pedagógica y los autores de manera conjunta redactan los documentos para publicar en revistas de investigación de vanguardia mundial. El Observatorio acompaña a los autores en proyectos posteriores de investigación asociados al proyecto que se construyó en el Laboratorio. Probablemente algunos proyectos por su potencial o porque son muy amplios requieren de una segunda fase en su desarrollo, en tal caso el Laboratorio tiene como primicia que todo proyecto que se inicie debe finalizarse, así que posteriormente se hace la segunda fase, si se requiere, porque un principio fundamental de la innovación es que sea una realidad tangible.

Hoy en día el aprendizaje mediado con tecnología requiere de ambientes más inmersivos (alta interacción del usuario) que permitan evidenciar la adquisición de competencias por parte de los estudiantes. Por ello desde la década de los 80 's se empezó a hablar de simuladores (herramientas de software que emulan procesos reales) con los cuales los estudiantes se enfrentaran en ambientes simulados a situaciones de su quehacer profesional, esto le permite a un egresado prepararse mejor para el mundo laboral y ser capaz de dar respuesta a diversas situaciones.

Por otro lado, desde hace varias décadas en el mundo empresarial se está trabajando lo que se llamamos business games o juego de negocios, también en la parte comportamental se implementaron los serious games o juegos serios para emular ambientes cotidianos donde se quiere saber la respuesta del individuo. Estos juegos han sido trabajados en la educación durante mucho tiempo, sólo que ahora los podemos llevar con mayor facilidad a los ambientes digitales y ampliar su uso educativo.

Aquí se presentan seis experiencias iniciales que se desarrollaron durante el año 2017, cinco simuladores y un juego gerencial.

La primera de ellas es un software para el Diagnóstico empresarial que fortalece la calidad de los productos de consultoría entregados por los estudiantes de Administración de Empresas; a partir de la metodología de diagnóstico propuesta por Fred Davis se realiza un desarrollo de software que permite al estudiante aplicar el método a cualquier tipo de empresa.

El segundo proyecto es un Simulador de análisis prospectivo, para este diseño se hizo una revisión conceptual y metodológica del proceso de análisis 
prospectivo, al final se desarrolló una herramienta tecnológica que permite la identificación de escenarios futuros a partir del análisis de las tendencias clave de índole interno y externo que puedan afectar a una organización en un horizonte temporal de diez años.

\section{El tercer proyecto es un Simulador de gestión financiera para Administración}

Financiera centrado en el análisis de dicha área que conduzca a la acertada toma de decisiones. Es una herramienta pensada para los futuros financistas que se enfrentan a contextos reales. El objetivo es que los estudiantes puedan analizar diversas empresas del sector real, confrontando sus conocimientos con su entorno laboral.

El cuarto proyecto que se presenta es un Simulador de inversión en Renta Variable para el Mercado de Capitales. Aunque esta experiencia ya existe en el mercado, la innovación está en que la herramienta se adapta a las necesidades académicas y les permite a los estudiantes una experiencia de aprendizaje más estructurada que incluye competencias en la interpretación, diagnóstico y toma de decisiones de inversión en el mercado de renta variable.

La quinta experiencia de aprendizaje es el Simulador de evaluación financiera de proyectos, es el software que realiza la simulación de evaluación de un proyecto de inversión que le permite a los estudiantes construir un aprendizaje significativo al ser capaces de validar la viabilidad de un proyecto de inversión para una empresa.

El sexto y último proyecto es un Juego Gerencial que se enmarca en la categoría de juego serio. Consiste en un video juego interactivo que enfrenta al estudiante a decisiones gerenciales basándose en la metodología Balanced Scorecard. Según las decisiones que tome cada participante serán asignados puntajes acumulativos que afectan los indicadores de la compañía. Aquí el estudiante es un Chiefs Executive Officer (CEO) que se enfrenta a las decisiones gerenciales que marcan el futuro de la compañía.

En este libro encontrará todo el proceso, desde una perspectiva educativa, cómo se diseñó y estructuró la estrategia pedagógica para que desarrollara las diferentes competencias, y cómo combinó los saberes propios de cada uno de los módulos a los que se vincula. La mayoría de los proyectos son productos completamente nuevos en el ámbito educativo con las patentes para la Institución.

Es para los autores es un gusto presentar este documento de las experiencias pedagógicas que se están implementando actualmente en cada uno de los 
módulos o cursos virtuales a los que pertenecen, esperamos que sean un aporte significativo para nuestros estudiantes y en general para el ámbito académico.

\section{Atentamente,}

Los autores. 